\title{
LIFTING THEOREMS IN NONSTANDARD MEASURE THEORY
}

\author{
DAVID ROSS
}

(Communicated by Andreas R. Blass)

\begin{abstract}
A nonstandard capacity construction, analogous to Loeb's measure construction, is developed. Using this construction and Choquet's Capacitability theorem, it is proved that a Loeb measurable function into a general (not necessarily second countable) space has a lifting precisely when its graph is 'almost' analytic. This characterization is used to generalize and simplify some known lifting existence theorems.

2. The standard notion of 'Lusin measurability' is related to the nonstandard notion of admitting a 'two-legged' lifting. An immediate consequence is a new and simple proof of the general Lusin theorem. Another consequence is the existence of a Loeb measurable function, not admitting a lifting, into a relatively small topological space.
\end{abstract}

\section{INTRODUCTION}

A fundamental tool in nonstandard measure theory is the 'lifting' construction, in which an (external) measurable function is approximated by an internal one. Most lifting theorems in the literature derive from a result of Anderson, which assumes that the target space is second countable.

Nonstandard measure theory is being used to study increasingly exotic structures (random sets, random measures, stochastic processes on infinite-dimensional Banach spaces), where the target spaces for measurable functions are not necessarily second countable (or even topological!), and more general lifting theorems are therefore required. It is natural to conjecture that any measurable function will have a lifting, provided the cardinality of the target space is small relative to the degree of saturation of the nonstandard model. This conjecture turns out to be false (Corollary 6.3 and Example 6.4).

The paper reduces questions about a function's liftability to questions about the analytic structure (in the sense of descriptive set theory) of the function's graph. The main tool used is Choquet's theorem from the theory of capacities; this theory is reviewed in $\S 2$, where a nonstandard capacity constructionanalogous to Loeb's measure construction-is described.

Received by the editors April 27, 1989 and, in revised form, September 27, 1989.

1980 Mathematics Subject Classification (1985 Revision). Primary 03H05, 28E05; Secondary 28A20, 28A05.

Research was supported by an SERC (U.K.) grant at Hull University. 
In $\S 3$ the main theorem is proved: a function has a lifting precisely when its graph is "almost" analytic. Some immediate consequences appear in $\S 4-a$ new proof of Anderson's result, and extensions to general metric spaces.

Sufficient conditions on the target space are considered in $\S 5$. Suppose, for example, that the target space $Y$ is not second countable, but has a coarser second countable Hausdorff topology. Anderson's theorem cannot automatically be applied, since $N S\left({ }^{*} Y\right)$ under the coarse topology might be much larger than $N S\left({ }^{*} Y\right)$ in the original topology. If, however, there is a set $Y^{\prime}$, analytic over the internals, with $Y \subseteq Y^{\prime} \subseteq N S\left({ }^{*} Y\right)$ (in the original topology) then the function has a lifting.

The standard property of being 'Lusin measurable' is related in $\S 6$ to the nonstandard property of admitting a 'two-legged' lifting. An immediate consequence is a nonstandard proof of the general Lusin theorem; another is existence, mentioned above, of a measurable function without a lifting.

\section{NONSTANDARD PRELIMINARIES}

I assume that the reader is familiar with nonstandard analysis at the level of [C1, C2, AFHL, HL, or SB]. All notation that is not common to the first four of these references will be defined anew.

It will be convenient to make the following assumption about the nonstandard model in which we work; namely, that for some cardinal $\kappa_{0} \geq \aleph_{1}$ :

(1) The model is $\kappa_{0}$-saturated.

(2) Every internal set has cardinality $\kappa_{0}$.

(3) $\kappa_{0}$ is not a (two-valued) measurable cardinal.

The requirements (2) and (3) on $\kappa_{0}$ are only applied to Theorem 4.2 and its consequences, therefore, in general, may be ignored if they offend. Models satisfying (1)-(3) can always be built.

\subsection{ANALYTIC SETS}

A pair $(X, \mathscr{K})$ is a paved space provided $X$ is a set and $\{\phi, X\} \subseteq \mathscr{K} \subseteq$ $\mathscr{P}(X)$; the family $\mathscr{K}$ is called a paving of $X$. The paving $\mathscr{K}$ is semicompact provided that whenever $\left\{K_{n}\right\}$ is a countable subset of $\mathscr{K}$ with $\bigcap_{n} K_{n}=\phi$. $\bigcap_{n<N} K_{n}=\phi$ for some finite $N$.

A $\mathscr{K}$-Suslin scheme is a subset $\left\{K_{\tau}\right\}$ of $\mathscr{K}$, where $\tau$ ranges over all finite sequences of natural numbers. If $f: \mathbb{N} \rightarrow \mathbb{N}$ and $n \in \mathbb{N}$, write $f \mid n$ for the sequence $\{f(0), \ldots, f(n)\}$. The kernel of the Suslin scheme $\left\{K_{\tau}\right\}$ is the set $\cup \cap K_{f \mid n}$, where the union ranges over functions $f: \mathbb{N} \rightarrow \mathbb{N}$ and the intersection ranges over $n \in \mathbb{N}$. Write $\mathscr{S}(\mathscr{K})$ for the collection of kernels of $\mathscr{K}$-Suslin schemes.

The next lemma summarizes some well-known results about Suslin schemes. 
2.1. Lemma. Let $(X, \mathscr{K})$ be a paved space, and let $\mathscr{B}(\mathscr{K})$ be the smallest sigma-algebra containing $\mathscr{K}$. Then:

(1) $\mathscr{S}(\mathscr{S}(\mathscr{K}))=\mathscr{S}(\mathscr{K})$,

(2) $\mathscr{B}(\mathscr{K}) \subseteq \mathscr{S}(\mathscr{K})$ if and only if $\left\{K^{c}: K \in \mathscr{K}\right\} \subseteq \mathscr{S}(\mathscr{K})$.

\subsection{CAPACITIES}

Let $\mathscr{K}$ be a regular paving of $X$, that is, $\mathscr{K}$ is closed under finite unions and intersections. A function $T: \mathscr{P}(X) \rightarrow[-\infty, \infty]$ is a $\mathscr{K}$-capacity on $X$ provided it is monotone nondecreasing (i.e., $A \subseteq B \Rightarrow T(A) \leq T(B)$ ) and it satisfies the following two continuity conditions:

(a) If $A_{0} \subseteq A_{1} \subseteq \cdots$ then $T\left(\bigcup_{n} A_{n}\right)=\sup _{n} T\left(A_{n}\right)$.

(b) If $A_{0} \supseteq A_{1} \supseteq \cdots$ are elements of $\mathscr{K}$ then $T\left(\bigcap_{n} A_{n}\right)=\inf _{n} T\left(A_{n}\right)$.

Capacities in this paper will invariably be finite (i.e., $\operatorname{range}(T) \subseteq \mathbb{R}$ ), though all the standard results and most of the nonstandard ones hold for the general case. The first result is due to Choquet.

2.2. Theorem. Let $T$ be a $\mathscr{K}$-capacity on $X$, where $\mathscr{K}$ is a regular paving of $X$, and let $A \in \mathscr{S}(\mathscr{K})$. Then $T(A)=\sup _{E \subseteq A, E \in \mathscr{K}_{\delta}} T(E)$, where $\mathscr{K}_{\delta}$ is the closure of $\mathscr{K}$ under countable intersections.

Again, let $\mathscr{K}$ be a regular paving of $X$. A monotone nondecreasing function $T: \mathscr{K} \rightarrow \mathbb{R}$ is $\mathscr{K}$-strongly subadditive provided

$$
T(A \cup B)+T(A \cap B) \leq T(A)+T(B)
$$

for every $A, B \in \mathscr{K}$. (If $\mathscr{K}=\mathscr{P}(X)$, just call $T$ strongly subadditive.)

The reader is warned that strong subadditivity is an asymmetric notion: If $T: \mathscr{K} \rightarrow[0, \infty)$ is strongly subadditive, and $T(A)=T(B)=0$ then $T(A \cup B)$ $=0$, but if $T(A)=T(B)>0$, and $B \subseteq A$, then $T(A-B)$ need not equal zero.

A $\mathscr{K}$-strongly subadditive function $T: \mathscr{K} \rightarrow[0, \infty)$ is a precapacity. Given such a $T$, define a function $L(T): \mathscr{P}(X) \rightarrow[0, \infty)$ by

$$
L(T)(E)=\inf _{\substack{E \subseteq D \\ D \in \mathscr{Z}_{\sigma}}} \sup _{K \in \mathbb{K} \in \mathbb{K}} T(K)
$$

where $\mathscr{K}_{\sigma}$ is the closure of $\mathscr{K}$ under countable unions. (The $L(T)$ notation is chosen to be consistent with the usual, if cumbersome, notation from nonstandard measure theory.)

2.3. Theorem. Let $\mathscr{K}$ be a regular paving of $X$ and $T$ be a precapacity on $\mathscr{K}$. Suppose that the following two continuity conditions are satisfied:

(a) If $A_{0} \subseteq A_{1} \subseteq \cdots$ are elements of $\mathscr{K}$ with $\bigcup_{n} A_{n} \in \mathscr{K}$ then $T\left(\bigcup_{n} A_{n}\right)=$ $\sup _{n} T\left(A_{n}\right)$.

(b) If $A_{0} \supseteq A_{1} \supseteq \cdots$ are elements of $\mathscr{K}$ then $L(T)\left(\bigcap_{n} A_{n}\right)=\inf _{n} T\left(A_{n}\right)$.

Then $L(T)$ is a strongly subadditive $\mathscr{K}$-capacity on $X$. 
Proof. This a weak form of [DM, Theorem 32].

Note (for later reference) under the conditions of Theorem 2.3, that if $A, B \subseteq X$ then strong subadditivity implies that $L(T)(A) \leq L(T)(A \cup B) \leq$ $L(T)(A-B)+L(T)(B)$; just replace $A$ by $A-B$ in the inequality defining strong subadditivity.

The next notation and propositions will be useful in subsequent sections. Suppose $\mathscr{K}$ is a regular paving of $X, T$ a precapacity on $\mathscr{K}$, and $X^{\prime}$ is another set. Let $\widehat{\mathscr{K}}$ be any regular paving of $X \otimes X^{\prime}$ such that for each $K \in$ $\widehat{\mathscr{K}}$, the projection $\operatorname{proj}_{X} K$ of $K$ onto $X$ is in $\mathscr{K}$. For $K \in \widehat{\mathscr{K}}$ define $\widehat{T}(K)=T\left(\operatorname{proj}_{X} K\right)$.

2.4. Proposition. $\widehat{T}$ is a $\widehat{\mathscr{K}}$-precapacity on $X \otimes X^{\prime}$.

Proof. It suffices to show that $\widehat{T}$ is strongly subadditive; but this follows immediately from the relations $\operatorname{proj}_{X}(A \cap B) \subseteq\left(\operatorname{proj}_{X} A\right) \cap\left(\operatorname{proj}_{X} B\right)$ and $\operatorname{proj}_{X}(A \cup B)$ $=\left(\operatorname{proj}_{X} A\right) \cup\left(\operatorname{proj}_{X} B\right)$.

2.5. Lemma. Suppose, with the above notation, that $E \otimes X^{\prime} \in \widehat{\mathscr{K}}$ whenever $E$ $\in \mathscr{K}$. If $F \subseteq X \otimes X^{\prime}, E=\operatorname{proj}_{X} F$, and $G=E \otimes X^{\prime}$, then $L(T)(E)=L(\widehat{T})(F)$ $=L(T)(G)$.

Proof. First, suppose $F \in \widehat{\mathscr{K}}_{\sigma}$. Observe that $E \in \mathscr{K}_{\sigma}$ and $G \in \widehat{\mathscr{K}}_{\sigma}$. By regularity of the pavings and the definition of $L(T)$, find sets $E_{n} \in \mathscr{K}, F_{n}, G_{n} \in \widehat{\mathscr{K}}$, with $E=\bigcup_{n} E_{n}, F=\bigcup_{n} F_{n}, G=\bigcup_{n} G_{n}$, and $L(T)(E)=\sup _{n} T\left(E_{n}\right)$, $L(\widehat{T})(F)=\sup _{n} \widehat{T}\left(F_{n}\right)$, and $L(\widehat{T})(G)=\sup _{n} \widehat{T}\left(G_{n}\right)$. Put $E_{n}^{\prime}=E_{n} \cup \operatorname{proj}_{X} G_{n}$, $G_{n}^{\prime}=E_{n}^{\prime} \otimes X^{\prime}$, and $F_{n}^{\prime}=F_{n} \cup\left(F \cap G_{n}^{\prime}\right)$; note $E_{n} \subseteq E_{n}^{\prime} \subseteq E, F_{n} \subseteq F_{n}^{\prime} \subseteq F$, and $G_{n} \subseteq G_{n}^{\prime} \subseteq G$. Since $E_{n}^{\prime} \subseteq \operatorname{proj}_{X} F_{n}^{\prime}, T\left(E_{n}^{\prime}\right)=\hat{T}\left(G_{n}^{\prime}\right) \leq \widehat{T}\left(F_{n}^{\prime}\right)$. Let $n \rightarrow \infty$ to obtain $L(\widehat{T})(E)=L(T)(G) \leq L(\widehat{T})(F)$; by monotonicity of $L(\widehat{T})$, $L(\widehat{T})(F) \leq L(\widehat{T})(G)$, completing the proof in this case.

Now, suppose $F$ is arbitrary; let $\left\{F_{n}\right\} \subseteq \widehat{\mathscr{K}}_{\sigma}$ with $F \subseteq \cdots \subseteq F_{2} \subseteq F_{1} \subseteq F_{0}$. Let $E_{n}=\operatorname{proj}_{X} F_{n}$, and note that $E_{n} \in \mathscr{K}_{\sigma} . L(T)(E) \leq \inf _{n} L(T)\left(E_{n}\right)=$ $\inf _{n} L(\widehat{T})\left(F_{n}\right)$, so $L(T)(E) \leq L(\widehat{T})(F)$. As before, $L(\widehat{T})(F) \leq L(\widehat{T})(G)$. It remains to show that $L(\widehat{T})(G) \leq L(T)(E)$.

Let $\left\{E_{n}\right\} \subseteq \mathscr{K}_{\sigma}$ with $E \subseteq \cdots \subseteq E_{2} \subseteq E_{1} \subseteq E_{0}$. Put $G_{n}=E_{n} \otimes X^{\prime}$, note $G_{n} \in \widehat{\mathscr{K}}_{\sigma}$. Then $L(\widehat{T})(G) \leq \inf _{n} L(\widehat{T})\left(G_{n}\right)=\inf _{n} L(T)\left(E_{n}\right)$, so $L(\widehat{T})(G) \leq$ $L(T)(E)$, which completes the proof.

\subsection{NONSTANDARD CAPACITIES}

$\Omega$ will adways denote an internal subset of the nonstandard universe, and $\mathscr{K} \subseteq \mathscr{P}(\Omega)$ will be a regular paving of $\Omega$. Note that in the next theorem, no internality constraints are put on $T$.

2.6. Theorem. Suppose $T$ is a precapacity on $\mathscr{K}$; then $L(T)$, as defined in $\S 2.2$, is a strongly subadditive $\mathscr{K}$-capacity on $\Omega$. 
Proof. It suffices to verify the two continuity conditions of Theorem 2.3. Condition (a) follows immediately by $\kappa_{0}$-saturation and the hypothesis that $\mathscr{K} \subseteq$ * $\mathscr{P}(\Omega)$. To verify condition (b), let $A_{0} \supseteq A_{1} \supseteq \cdots$ be elements of $\mathscr{K}$, and let $A=\bigcap_{n} A_{n}$. Clearly $L(T)$ is monotone nondecreasing, so $L(T)(A) \leq$ $\inf _{n} T\left(A_{n}\right)$. For the other inequality, suppose $A \subseteq D \in \mathscr{K}_{\sigma}$. Since $\mathscr{K}$ is regular, $D=\bigcup_{n} D_{n}$ for some sequence $D_{0} \subseteq D_{1} \subseteq \cdots$ with each $D_{n} \in \mathscr{K}$. By saturation, $\bigcap_{n} A_{n} \subseteq \bigcup_{n} D_{n}$ implies that for some $m, A_{m} \subseteq D_{m}$. It follows that $T\left(A_{m}\right) \leq T\left(D_{m}\right) \leq \sup _{K \subseteq D, K \in \mathscr{K}} T(K)=L(T)(D)$, so $\inf _{n} T\left(A_{n}\right) \leq L(T)(A)$ as required.

Call a precapacity $T$ on $\mathscr{K}$ internally generated provided $\mathscr{K}$ is internal and there is an internal $T^{\prime}: \mathscr{K} \rightarrow^{*} \mathbb{R}$ with $T(E) \approx T^{\prime}(E)$ for every $E \in \mathscr{K}$. Choquet's Theorem 2.2 has a strong form for capacities $L(T)$ with $T$ internally generated.

2.7. Theorem. Suppose $T$ is an internally generated precapacity on internal $\mathscr{K}$ and $A \in \mathscr{S}(\mathscr{K}) ;$ then $L(T)(A)=\sup _{E \subseteq A, E \in \mathscr{K}} T(E)$.

Proof. Let $A \in \mathscr{S}(\mathscr{K}), r<L(T)(A)$, and (by Theorem 2.2) $\left\{E_{n}\right\} \subseteq \mathscr{K}$ with $L(T)\left(\bigcap_{n} E_{n}\right)>r$ and $\bigcap_{n} E_{n} \subseteq A$. Let $T^{\prime}$ internal with $T^{\prime} \approx T$ on $\mathscr{K}$. By $\kappa_{0}-$ saturation there is an $E \in \mathscr{K}$ with $E \subseteq \bigcap_{n} E_{n}$ and $T^{\prime}(E) \gtrsim r$, so $T(E) \geq r$; this proves the result.

To put this result in perspective, the definition of $L(T)$ and Choquet's theorem together imply that for each $A \in \mathscr{S}(\mathscr{K})$ there is a $C \in \mathscr{K}_{\delta \sigma}$ and a $B \in \mathscr{K}_{\sigma \delta}$ with $C \subseteq A \subseteq B$ and $L(T)(C)=L(T)(A)=L(T)(B)$. (The set $B$ exists for arbitrary $A$.) Theorem 2.7 says that we may take $C \in \mathscr{K}_{\sigma}$ when $T$ is internally generated. A similar argument shows that we may take $B \in \mathscr{K}_{\delta}$ as well.

An important special case of these ideas is Peter Loeb's measure construction. Suppose $(\Omega, \mathscr{A}, \mu)$ is an internal finitely-additive probability; then ${ }^{\circ} \mu$ is an internally-generated precapacity, and the restriction of $L(\mu) \equiv L\left({ }^{\circ} \mu\right)$ to $L(\mathscr{A})$, the smallest sigma-algebra containing $\mathscr{A}$, is a countably-additive measure which is inner regular with respect to elements of $\mathscr{A}$. A straightforward saturation argument now proves the following.

2.8. Proposition. Suppose $(\Omega, \mathscr{A}, \mu)$ is an internal finitely-additive probability, and $L(\mathscr{A})$ is the smallest $\sigma$-algebra containing $\mathscr{A}$. For every $E \in L(\mathscr{A})$ there is some $A \in \mathscr{A}$ with $L(\mu)(E \Delta A)=0$.

\section{LifTings}

Let $Z$ be an internal set, $Z_{0}$ a (possibly external) subset of $Z, Y$ an arbitrary set, and $\phi$ a function from $Z_{0}$ onto $Y$. Usually $Y$ will be a Hausdorff topological space with $2^{\operatorname{card}(Y)}<\kappa_{0}, Z={ }^{*} Y, Z_{0}=N S\left({ }^{*} Y\right)$, and $\phi$ the standard part map. The more general case is useful, however, as will be seen in $\S \S 4$ and 5 . 
Let $\mathscr{K}$ be a regular paving of $\Omega$, closed under complements, $T$ an internally generated precapacity on $\mathscr{K}$, and $f: \Omega \rightarrow Y$ an arbitrary function. A function $F: \Omega \rightarrow Z$ is a lifting of $f$ provided $F$ is internal and the following diagram commutes off a set of capacity zero,

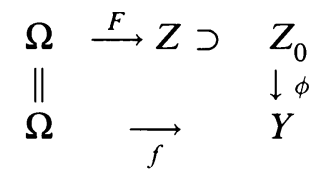

that is, $L(T)(\{\omega \in \Omega: f(\omega) \neq \phi(F(\omega))\})=0$.

Denote by $\Gamma(f)$ the graph of $f, \Gamma(f)=\{(\omega, y): f(\omega)=y\}$. Let $\Gamma^{\circ}(f)=$ $\left\{(\omega, z) \in \Omega \otimes Z_{0}: f(\omega)=\phi(z)\right\}$.

If $(\omega, z) \in \Omega \otimes Z_{0}$ let $\phi(\omega, z)=(\omega, \phi(z)) \in \Omega \otimes Y$. Put

$$
\begin{aligned}
& \mathscr{K}_{0}=\left\{E \in \mathscr{P}^{*}(\Omega \otimes Z): \operatorname{proj}_{\Omega} E \in \mathscr{K}\right\}, \\
& \mathscr{K}_{1}=\left\{E \in \mathscr{K}_{0}: E \subseteq \Omega \otimes Z_{0}\right\}, \text { and } \\
& \mathscr{K}_{2}=\left\{\phi(E): E \in \mathscr{K}_{1}\right\} .
\end{aligned}
$$

Call a set $E$ almost $\mathscr{S}\left(\mathscr{K}_{i}\right), i=0,1,2$, provided that for some $D \in \mathscr{S}\left(\mathscr{K}_{i}\right)$, $L(T)\left(\operatorname{proj}_{\Omega}(D \Delta E)\right)=0 . E$ has an $\mathscr{S}\left(\mathscr{K}_{i}\right)$-almost section if for some $D \in$ $\mathscr{S}\left(\mathscr{K}_{i}\right), L(T)\left(\operatorname{proj}_{\Omega}(D-E)\right)=L(T)\left(\operatorname{proj}_{\Omega} E-\operatorname{proj}_{\Omega} D\right)=0$.

The main result can now be stated.

3.1. Theorem. Suppose $f: \Omega \rightarrow Y$; then $a \Rightarrow b \Rightarrow c \Rightarrow d$, where :

(a) $f$ has a lifting,

(b) $\Gamma(f)$ is almost $\mathscr{S}\left(\mathscr{K}_{2}\right)$,

(c) $\Gamma^{\circ}(f)$ has an $\mathscr{S}\left(\mathscr{K}_{0}\right)$-almost section,

(d) $\exists F: \Omega \rightarrow Z$ internal with $L(T)(\{\omega: f(\omega)=\phi(F(\omega))\})=L(T)(\Omega)$.

Moreover, if $T$ is finitely additive then $(\mathrm{a})-(\mathrm{d})$ are equivalent.

Proof. The last remark follows from $a \Rightarrow b \Rightarrow c \Rightarrow d$, since if $T$ is finitely additive then so is $L(T)$.

$(\mathrm{a} \Rightarrow \mathrm{b})$ Let $F$ lift $f$, find $A \subseteq \Omega$ with $L(T)(A)=0$ and $f=\phi \circ F$ on $\Omega-A$. By the remarks following Theorem 2.7, we may assume that $A \in \mathscr{K}_{\delta}$, and so find $\left\{A_{n}\right\} \subseteq \mathscr{K}$ with $A \subseteq \cdots \subseteq A_{2} \subseteq A_{1} \subseteq A_{0}$ and $A=\bigcap_{n} A_{n}$. Let $F_{n}=\left\{(\omega, F(\omega)): \omega \in \Omega-A_{n}\right\} \in \mathscr{K}_{1}$, put $D_{n}=\phi\left(F_{n}\right)$, observe that $D_{n} \subseteq \Gamma(f)$ and $\operatorname{proj}_{\Omega} D_{n}=\Omega-A_{n}$, and let $D=\bigcup_{n} D_{n}$. Then $D \subseteq \Gamma(f)$ and $\operatorname{proj}_{\Omega}(\Gamma(f)-D)=A$, so $D \in\left(\mathscr{K}_{2}\right)_{\sigma} \subseteq \mathscr{S}\left(\mathscr{K}_{2}\right)$ and $L(T)\left(\operatorname{proj}_{\Omega}(D \Delta \Gamma(f))\right)=0$; this proves $(\mathrm{b})$.

$(\mathrm{b} \Rightarrow \mathrm{c})$ Let $E \in \mathscr{S}\left(\mathscr{K}_{2}\right)$ with $L(T)(A)=0$, where $A=\operatorname{proj}_{\Omega}(E \Delta \Gamma(f))$. Write $E=\cup \cap E_{g \mid n}$ with $E_{g \mid n} \in \mathscr{K}_{2}$, and for $\tau$ a finite sequence of natural numbers find $D_{\tau} \in \mathscr{K}_{1}$ with $E_{\tau}=\phi\left(D_{\tau}\right)$. Put $D=\cup \cap D_{g \mid n}$. Let $(\omega, z) \in D$; since $D \in \mathscr{S}\left(\mathscr{K}_{1}\right), z \in Z_{0}$, and $(\omega, \phi(z)) \in \phi(D) \subseteq E$. If $\omega \notin A$ then $(\omega, \phi(z)) \in \Gamma(f)$ so $(\omega, z) \in \Gamma^{\circ}(f)$. It follows that $\operatorname{proj}_{\Omega}\left(D-\Gamma^{\circ}(f)\right) \subseteq A$, so $L(T)\left(\operatorname{proj}_{\Omega}\left(D-\Gamma^{\circ}(f)\right) \leq L(T)(A)=0\right.$. Moreover, $\operatorname{proj}_{\Omega} D \supseteq \operatorname{proj}_{\Omega} E \supseteq$ $\left(\operatorname{proj}_{\Omega} \Gamma(f)\right)-A$, so $L(T)\left(\Omega-\operatorname{proj}_{\Omega} D\right)=0$, proving (c). 
(c $\Rightarrow$ d) Let $T_{0}$ be the $\mathscr{K}_{0}$ precapacity induced by $T$ as in Proposition 2.4. By (c) and Theorem 2.7 find a sequence $D_{1} \subseteq D_{2} \subseteq \cdots \subseteq D, D_{n} \in \mathscr{K}_{0}$, with $L\left(T_{0}\right)\left(D_{n}\right) \rightarrow L\left(T_{0}\right)(D)$. By saturation find an internal $F: \Omega \rightarrow Z$ satisfying $\forall \omega \in \operatorname{proj}_{\Omega} D_{n},(\omega, F(\omega)) \in D_{n}$ for each $n \in \mathbb{N}$. Then $\phi(F(\omega))=f(\omega)$ for all $\omega \in \operatorname{proj}_{\Omega}\left(\bigcup_{n} D_{n}\right)-\operatorname{proj}_{\Omega}\left(D-\Gamma^{\circ}(f)\right)$. Thus,

$$
\begin{aligned}
L(T)(\{\omega: f(\omega)=\phi(F(\omega))\} & \geq L(T)\left(\operatorname{proj}_{\Omega} \bigcup_{n} D_{n}-\operatorname{proj}_{\Omega}\left(D-\Gamma^{\circ}(f)\right)\right) \\
& =L(T)\left(\operatorname{proj}_{\Omega} \bigcup_{n} D_{n}\right) \quad \text { by strong subadditivity } \\
& =L\left(T_{0}\right)\left(\bigcup_{n} D_{n}\right) \quad \text { by Lemma } 2.5 \\
& =L\left(T_{0}\right)(D) \\
& =L(T)\left(\operatorname{proj}_{\Omega} D\right) \\
& =L(T)(\Omega) \quad\left(\text { since } L(T)\left(\Omega-\operatorname{proj}_{\Omega} D\right)=0\right) .
\end{aligned}
$$

This proves (d), and the theorem.

\section{APPLications to MEASURABle FUnCtions}

The remainder of this paper considers only Loeb probability measures, i.e. probability measures $(\Omega, L(\mathscr{A}), L(\mu))$ obtained from internal probability measures $(\Omega, \mathscr{A}, \mu)$ as in Proposition 2.8. Many of the results remain true for arbitrary capacities, but definitions and theorem statements become unwieldy.

In this section I consider some straightforward applications of $(c \Rightarrow a)$ of Theorem 3.1. Note that by the remarks following Theorem 2.7, every set of capacity zero is contained in a set which is Loeb measurable with Loeb measure zero. It follows that the liftings produced here are the same as those which usually appear in the theory of Loeb measures.

Let $\mathscr{C}$ be a paving of $Y$, and $f: \Omega \rightarrow Y$; then $f$ is $(\mathscr{C}-)$ measurable if $f^{-1}(C) \in L(\mathscr{A})$ whenever $C \in \mathscr{C}$. Call $\mathscr{C}$ basis-like if there is a function ${ }^{\sharp}: \mathscr{C} \rightarrow{ }^{*} \mathscr{P}(Z)$ such that for every $y \in Y, \varnothing \neq \cap\left\{{ }^{\sharp} C: y \in C \in \mathscr{C}\right\} \subseteq \phi^{-1}(y)$. The following result, when $\mathscr{C}$ is a countable basis for a Hausdorff space, is due to Anderson.

4.1. Theorem. Suppose $\mathscr{C}$ is basis-like and countable, and $f: \Omega \rightarrow Y$ is measurable. Then $f$ has a lifting.

Proof. Let $\mathscr{C}=\left\{C_{n}: n \in \mathbb{N}\right\}$. 'For each $n$ take $B_{n} \in \mathscr{A}$ so that $A_{n}=$ $f^{-1}\left(C_{n}\right) \Delta B_{n}$ has $L(\mu)$-measure zero. Put $D=\bigcap_{n}\left(B_{n} \otimes^{\sharp} C_{n}\right) \cup\left(B_{n}^{c} \otimes Z\right) \in \mathscr{S}\left(\mathscr{K}_{0}\right)$, and put $A=\bigcup_{n} A_{n}$. If $\omega \in \Omega-A$ then $\{z \in Z:(\omega, z) \in D\} \subseteq \cap\left\{{ }^{\sharp} C: f(\omega) \in\right.$ $C \in \mathscr{C}\} \subseteq \phi^{-1}(f(\omega))$, so $L(\mu)\left(\operatorname{proj}_{\Omega}\left(D-\Gamma^{\circ}(f)\right)\right) \leq L(\mu)(A)=0$. If $\omega \notin A$ then $\varnothing \neq \cap\left\{{ }^{\sharp} C: f(\omega) \in C\right\} \subseteq \cap\left\{{ }^{\sharp} C_{n}: \omega \in B_{n}\right\} \subseteq D$, so $L(\mu)\left(\operatorname{proj}_{\Omega}\left(\Gamma^{\circ}(f)\right)-\right.$ $\left.\operatorname{proj}_{\Omega}(D)\right)=L(\mu)\left(\operatorname{proj}_{\Omega}(D)^{c}\right) \leq L(\mu)(A)=0$. $D$ is thus an $\mathscr{S}\left(\mathscr{K}_{0}\right)$-section of $\Gamma^{\circ}(f)$; it follows from Theorem 3.1 that $f$ has a lifting. 
The most straightforward application of this result is the one originally proved by Anderson, that is, the case where $\mathscr{E}$ is a countable basis for a Hausdorff space $Y, Z={ }^{*} Y, Z_{0}=N S\left({ }^{*} Y\right)$, and $\phi$ is the standard part map. I'll now consider a more interesting family of examples, the so-called nonstandard hulls, where $Y$ need not be a standard object and where the natural paving of $Y$ is not a priori countable.

The next theorem is the only place in this paper where the peculiar conditions on the nonstandard model (i.e. that $\kappa_{0}$ not be measurable) are required. The reader is referred to [R1] for the proof, which is rather cumbersome; the idea is from $[\mathrm{F}]$.

4.2. Theorem. Suppose $Y$ is a metric space, and $f: \Omega \rightarrow Y$ is measurable. (Here $\mathscr{C}$ consists of all open subsets of $Y$.) Then $f\left(\Omega^{\prime}\right)$ is separable for some $\Omega^{\prime} \subseteq \Omega$ with $L(\mu)\left(\Omega^{\prime}\right)=1$.

Given a general quadruple $\left(Z, Z_{0}, Y, \phi\right)$ and a paving $\mathscr{C}$ of $Y$, say $\mathscr{C}$ is very basis-like provided there is a function ${ }^{\sharp}: \mathscr{C} \rightarrow^{*} \mathscr{P}(Z)$ satisfying

(i) $\forall C \in \mathscr{C}, \phi^{-1}(C) \subseteq{ }^{\sharp} C$; and

(ii) $\forall z_{0} \in Z_{0} \quad \forall z \in Z-\phi^{-1}\left(\phi\left(Z_{0}\right)\right) \exists C \in \mathscr{C},\left(z_{0}, z\right) \in{ }^{\sharp} C \otimes\left({ }^{\sharp} C\right)^{c}$ and $\phi\left(z_{0}\right) \in C$.

4.3. Lemma. If $\mathscr{C}$ is very basis-like then $\mathscr{C}$ is basis-like; in fact, $\phi^{-1}(y)=$ $\cap\left\{{ }^{\sharp} C: y \in C \in \mathscr{C}\right\}$.

Proof. Let ${ }^{\sharp}$ be the function satisfying (i) and (ii). Let $y \in Y, A=\cap\left\{{ }^{\sharp} C: y \in\right.$ $C \in \mathscr{C}\}$, and $z_{0} \in \phi^{-1}(y)$ (which $\subseteq A$, by i). Let $z \in A$. If $z \notin \phi^{-1}(y)$ then by (ii) there is a $C \in \mathscr{C}$ with $z_{0} \in{ }^{\sharp} C, z \notin{ }^{\sharp} C$, and $y \in C$, so $z \in A \subseteq{ }^{\sharp} C$, a contradiction. Thus $A \subseteq \phi^{-1}(y)$. For the opposite inclusion, suppose $z \in \phi^{-1}(y)$. If $y \in C \in \mathscr{C}$ then (by (i)) $z \in \phi^{-1}(C) \subseteq{ }^{\sharp} C$, so $z \in A$. This proves the lemma.

4.4. Example (nonstandard hulls). Let $(Z, \rho)$ be an internal metric space, let $z_{0} \in Z$, let $Z_{1}=\left\{z \in Z: \rho\left(z, z_{0}\right)\right.$ is finite $\}$, let $Y_{1}=Z_{1} / \sim$ where $z_{1} \sim z_{2}$ provided $\rho\left(z_{1}, z_{2}\right) \approx 0$, and let $\phi$ be the quotient map from $Z_{1}$ onto $Y_{1}$. It is easy to see that the function $d: Y^{2} \rightarrow[0, \infty)$, defined by $d(x, y)={ }^{\circ} \rho\left(x^{\prime}, y^{\prime}\right)$, where $\phi\left(x^{\prime}\right)=x, \phi\left(y^{\prime}\right)=y$, is well defined and a metric on $Y$. The space $Y$ is called a nonstandard hull.

Suppose $f: \Omega \rightarrow Y$ is measurable (in the sense of the metric topology on $Y)$. By Theorem 4.2, we may suppose that $Y=\operatorname{range}(f)$ is separable. Put $Z_{0}=\phi^{-1}(Y)$, and let $Y_{0}$ be a countable dense subset of $Y$. Let $\mathscr{C}$ be the collection of open sets of the form $N(y, n)=\left\{y^{\prime} \in Y: d\left(y, y^{\prime}\right)<1 / n\right\}$ where $y \in Y_{0}$ and $n \in \mathbb{N}$. If $C=N(y, n) \in \mathscr{C}$ let ${ }^{\sharp} C=\{z \in Z: \rho(z, \eta(y))<2 / n\}$, where $\eta: Y \rightarrow Z_{0}$ is any (fixed) inverse of $\phi$.

The function \# satisfies the defining conditions for $C$ to be very basis-like, so $\mathscr{C}$ is basis-like, and $f$ has a lifting. 


\section{SUFFICIENT CONDITIONS}

I now consider conditions on $Y$ sufficient to ensure that measurable functions into $Y$ have liftings.

Let $\mathscr{C}$ be a paving of $Y$, and $\mathscr{C}_{0}=\mathscr{C} \otimes^{*} \mathscr{P}(Z)$. Let $D(Y)$ be the diagonal of $Y, D(Y)=\{(y, y): y \in Y\}$, and let $D^{\circ}(Y)=\left\{(y, z) \in Y \otimes Z_{0}: \phi(z)=y\right\}$. Call $Y \mathscr{C}$-diagonalizable provided that for some $E \in \mathscr{S}\left(\mathscr{C}_{0}\right), D(Y) \subseteq E \subseteq$ $D^{\circ}(Y)$. Note that if $Y$ is $\mathscr{C}$-diagonalizable then $Y \subset Z_{0}$ and $y=\phi(y)$ for all $y \in Y$.

5.1. Theorem. If $Y$ is a $\mathscr{C}$-diagonalizable, and $f: \Omega \rightarrow Y$ is $\mathscr{C}$-measurable, then $f$ has a lifting.

Proof. Define a function $\Psi: \Omega \otimes Z \rightarrow Y \otimes Z$ by $\Psi(\omega, z)=(f(\omega), z)$. Let $E=\cup \cap E_{g \mid n} \in \mathscr{S}\left(\mathscr{C}_{0}\right)$ with $D(Y) \subseteq E \subseteq D^{\circ}(Y)$. For $\tau$ a finite sequence of natural numbers let $C_{\tau} \in \mathscr{C}, B_{\tau} \in{ }^{*} \mathscr{P}(Z)$ with $E_{\tau}=C_{\tau} \otimes B_{\tau}$, and let $A_{\tau} \in \mathscr{A}$ with $L(\mu)\left(A_{\tau} \Delta f^{-1}\left(C_{\tau}\right)\right)=0$. Put $D=\cup \cap\left(A_{g \mid n} \otimes B_{g \mid n}\right)$, and observe that $D-(A \otimes Z) \subseteq \Psi^{-1}(E) \subseteq \Gamma^{\circ}(f)$ and $(\Omega-A) \subseteq \operatorname{proj}_{\Omega} D$, where $A=\cup\left\{A_{\tau} \Delta C_{\tau}: \tau\right.$ a finite sequence from $\mathbb{N}\}$. This last union is countable, so has measure zero. Condition (c) of Theorem 3.1 is satisfied, so $f$ has a lifting.

5.2. Example (cf. [AFHL, Proposition 4.3.13]). Suppose $\mathscr{V}$ and $\mathscr{W}$ are topological spaces; a function $\chi: \Omega \times \mathscr{V} \rightarrow \mathscr{W}$ is a (continuous-path) stochastic process provided $\chi(\omega, v)$ is a continuous function of $v$ for each $\omega$, and a measurable function of $\omega$ for each $v$. A uniform lifting of $\chi$ is an internal function $X: \Omega \otimes^{*} \mathscr{V} \rightarrow^{*} \mathscr{W}$ such that for some $\Omega^{\prime} \subseteq \Omega$ with $L(\mu)\left(\Omega^{\prime}\right)=1$, $X(\omega, v) \approx \chi\left(\omega,{ }^{\circ} v\right)$ for all $v \in N S\left({ }^{*} \mathscr{V}\right), \omega \in \Omega^{\prime}$.

Let $Y=C(\mathscr{V}, \mathscr{W})$, the set of continuous functions from $\mathscr{V}$ to $\mathscr{W}$, be given the point-open topology (where a subbasic open set is one of the form $\{f \in Y: f(x) \in \beta\}$, with $x \in \mathscr{V}$ and $\beta$ an open subset of $\mathscr{W})$. The function $\omega \mapsto x(\omega, \cdot)$ (which will, by abuse of notation, also be written $\chi: \Omega \rightarrow Y$ ) is measurable, and a lifting in the usual sense is a uniform lifting of the stochastic process. If $\mathscr{V}$ and $\mathscr{W}$ are separable metric spaces then there is a metrizable topology on $Y$, finer than the point-open topology but giving the same Borel structure, and the methods of $\S 4$ can be used to produce a uniform lifting. This is the case studied-using different methods-in [AFHL].

Suppose now only that $\mathscr{V}$ and $\mathscr{W}$ are second-countable, and that $\mathscr{W}$ is regular. While the proof in [AFHL] can with care be extended to this case, I'll show that $D^{\circ}(Y)$ is $\mathscr{C}$-diagonalizable, where $\mathscr{C}$ is the Borel sigma-algebra given by the point-open topology on $Y, Z=^{*} Y$, and $Z_{0}$ is the set of $h^{\prime} \in Z$ such that for some $h=\phi\left(h^{\prime}\right) \in Y, h\left({ }^{\circ} v\right) \approx h^{\prime}(v)$ for every $v \in N S\left({ }^{*} \mathscr{V}\right)$.

Let $\mathscr{V}_{0}=\left\{\alpha_{n}: n \in \mathbb{N}\right\}$ and $\mathscr{W}_{0}=\left\{\beta_{n}: n \in \mathbb{N}\right\}$ be bases for $\mathscr{V}$ and $\mathscr{W}$; without loss of generality, every element of $\mathscr{W}_{0}$ appears infinitely often in the enumeration. If $i, j \in \mathbb{N}$ let $C_{i j}=\left\{f \in Y: f\left(\alpha_{i}\right) \subseteq \bar{\beta}_{j}\right\}$, where $\bar{\beta}$ is the closure of $\beta$ in $\mathscr{W}$. Observe that $C_{i j}=\bigcap_{r}\left\{h \in Y: h(r) \in \beta_{j}\right\}$, where $r$ 
ranges over a countable dense subset of $\alpha_{i}$, so $C_{i j} \in \mathscr{C}$. Fix a bijection $\gamma$ between $\mathbb{N}$ and the finite subsets of $\mathbb{N}$, and if $\tau=\left(\tau_{0}, \ldots, \tau_{n}\right)$ is a finite sequence from $\mathbb{N}$ let

$$
A_{\tau}=\left(\bigcap_{i \in \gamma(\tau(n))} C_{i n}\right) \cap\left(\bigcap_{\substack{i \notin \gamma(\tau(n)) \\ i \leq n}} C_{i n}^{c}\right) .
$$

Now let $E=\cup \cap\left(A_{g \mid n} \otimes^{*} A_{g \mid n}\right)$; it remains to show that $D(Y) \subseteq E \subseteq D^{\circ}(Y)$. For the first inclusion, let $f \in Y$ and define $\sigma: \mathbb{N} \rightarrow \mathbb{N}$ by $\sigma(n)=\gamma^{-1}(\{i \leq$ $\left.\left.n: f\left(\alpha_{i}\right) \in \bar{\beta}_{n}\right\}\right)$. Then $f \in \bigcap_{n} A_{\sigma \mid n}$, so $D(Y) \subseteq E$.

Suppose now that $(f, F) \in \bigcap_{n}\left(A_{\sigma \mid n} \otimes^{*} A_{\sigma \mid n}\right)$, and $\phi(F) \neq f$. There then exists an $x \in N S\left({ }^{*} \mathscr{V}\right)$ with $f\left({ }^{\circ} x\right) \not F(x)$. For some $n \in \mathbb{N}, f\left({ }^{\circ} x\right) \in \beta_{n}$ but $F(x) \notin^{*} \beta_{n}$; since $\mathscr{W}$ is regular we may assume that $F(x) \notin^{*} \bar{\beta}_{n}$. Let $m \in \mathbb{N}$ with ${ }^{\circ} x \in \alpha_{m} \subseteq f^{-1}\left(\beta_{n}\right)$. Every element of $\mathscr{W}_{0}$ appears infinitely often in the enumeration, so without loss of generality $n>m$. It follows that $m \in \gamma(\sigma(n))$. Since $x \in \in^{*} \alpha_{m}$, and $F \in^{*} A_{\sigma \mid n}, F(x) \in F\left({ }^{*} \alpha_{m}\right) \subseteq^{*} \bar{\beta}_{n}$, a contradiction. This proves the second inclusion. Thus, $\chi$ has a uniform lifting.

It often happens that while the topology on a space $Y$ is not itself second countable, it has a second countable Hausdorff subtopology. Unfortunately, this subtopology will have more nearstandard points than the original one, so a lifting into $Y$ relative to it may not work for the original topology. The next results show that liftings can be obtained provided there aren't too many elements of $Y$ which are not nearstandard.

Call the quadruple $\left(Z, Z_{0}, Y, \phi\right)$ nearly standard provided there is some $E \in \mathscr{S}\left({ }^{*} \mathscr{P}(Z)\right)$ with $Y \subseteq E \subseteq Z_{0}$ and $\phi(E)=Y$.

5.3. Theorem. Let $\mathscr{C}$ be a paving of $Y, Z_{0}^{\prime} \subseteq Z$, and $\phi^{\prime}: Z_{0}^{\prime} \rightarrow Y$ such that $Z_{0} \subseteq Z_{0}^{\prime}$ and $\phi=\phi^{\prime}$ on $Z_{0}$. Suppose that $f: \Omega \rightarrow Y$ is $\mathscr{C}$-measurable, that $\left(Z, Z_{0}, Y, \phi\right)$ is nearly standard, and that $Y$ is $\mathscr{C}$-diagonalizable (in the sense of $\left.Z_{0}^{\prime}, \phi^{\prime}\right)$. Then $f$ has a lifting.

Proof. Let $E_{0} \in \mathscr{S}\left(\mathscr{C}_{0}\right)$ with $D(Y) \subseteq E_{0} \subseteq D^{\circ}(Y)^{\prime}$, where $D^{\circ}(Y)^{\prime}=\{(y, z) \in$ $\left.\left(Y \otimes Z_{0}^{\prime}\right): \phi^{\prime}(z)=y\right\}$. Let $E_{1} \in \mathscr{S}\left({ }^{*} \mathscr{P}(Z)\right)$ with $E_{1} \subseteq Z_{0}$ and $\phi\left(E_{1}\right)=Y$. Put $E=E_{0} \cap\left(Y \otimes E_{1}\right) \in \mathscr{S}\left(\mathscr{C}_{0}\right)$, and note $D(Y) \subseteq E \subseteq D^{\circ}(Y)^{\prime}$. If $(y, z) \in E$ then $\phi^{\prime}(z)=y$ and $z \in E_{1} \subseteq Z_{0}$, so $\phi(z)=y$, and $D(Y) \subseteq E \subseteq D^{\circ}(Y)$. The result now follows from Theorem 5.1.

5.4. Corollary. Suppose $(Y, \tau)$ is a sigma-compact topological space $\tau^{\prime} \subseteq \tau$ is countable, and the topology on $Y$ generated by $\tau^{\prime}$ is Hausdorff. Every $\tau^{\prime}$ measurable function into $Y$ has a lifting (with respect to topology $\tau$ ).

Proof. In this case, $Z={ }^{*} Y, Z_{0}=N S\left({ }^{*} Y\right)$, and $\phi$ is the standard part map. Let $Z_{0}^{\prime}$ be the $\tau^{\prime}$-nearstandard elements of ${ }^{*} Y$, and let $\phi^{\prime}$ be the standard part map relative to $\tau^{\prime}$; note that $\phi^{\prime}=\phi$ on $Z_{0}$. 
If $Y=\bigcup_{n} K_{n}, K_{n} \tau$-compact, then ${ }^{*} K_{n} \subseteq \phi^{-1}\left(K_{n}\right)$, and the set $E=\bigcup_{n}^{*} K_{n}$ shows that $\left({ }^{*} Y, N S\left({ }^{*} Y\right), Y\right.$, st $)$ is nearly standard. Evidently $D^{\circ}(Y)^{\prime}=$ $\bigcap_{u \in \tau^{\prime}}\left(u \otimes^{*} u\right) \cup\left(u^{c} \otimes Z\right)$, so $Y$ is $\tau^{\prime}$-diagonalizable. The conclusion follows from Corollary 5.3.

As noted above, the condition that $Y \subseteq Z_{0}$ is implicit in the definition of $\mathscr{C}$-diagonalizability. For more general quadruples $(Z, Z, Y, \phi)$ call $Y$ weakly $\mathscr{C}$-diagonalizable if for some $E \in \mathscr{S}\left(\mathscr{C}_{0}\right), E \subseteq D^{\circ}(Y)$ and $D(Y)=$ $\{(y, \phi(z)):(y, z) \in E\}$. It is not difficult to see that weak $\mathscr{C}$-diagonalizability need not imply $\mathscr{C}$-diagonalizability, even when $Y \subseteq Z_{0}$. However, the proof of Theorem 5.1 holds under the appropriate changes.

\section{LUSIN MEASURABILITY}

In this section, $X$ and $Y$ are Hausdorff topological spaces, $m$ is a probability measure on the Borel sigma-algebra $\mathscr{B}$ over $X, f: X \rightarrow Y$, and $(\Omega, L(\mathscr{A}), L(\mu))$ is a Loeb probability space with $\Omega \subseteq{ }^{*} X$, such that $(X, \mathscr{B}$, $m)$ is the image of $(\Omega, L(\mathscr{A}), L(\mu))$ under the standard part map. Observe that by [LR, Theorem 4] this necessarily means that $m$ is a Radon (i.e., compact-inner-regular) measure on $(X, \mathscr{B})$.

The results of earlier sections will be applied with $Z={ }^{*} Y, Z_{0}=N S\left({ }^{*} Y\right)$, and $\phi=$ st.

The function $f$ is Lusin measurable provided for every $\varepsilon>0$ there is a compact $K \subseteq X$ with $m(K)>1-\varepsilon$ such that the restriction of $f$ to $K$ is continuous. Such an $f$ is always measurable, and sometimes it is strongly Lusin measurable in the sense that for each $\varepsilon>0$ there is a continuous $g: X \rightarrow Y$ with $m(\{x: f(x) \neq g(x)\})<\varepsilon$. A well-known theorem of Lusin asserts that a measurable function from a Radon space into a second countable space is Lusin measurable; see also Corollary 6.2.

Say the function $f$ has a (two-legged) lifting provided the function $f \circ$ st: $\Omega \rightarrow Y$ has a lifting. (Strictly speaking, st, hence $f \circ$ st, need not be defined on all of $\Omega$; this is not important, as $L(\mu)\left(\mathrm{st}^{-1}(X) \cap \Omega\right)=1$, so we can define $f \circ$ st arbitrarily off $\Omega \cap N S\left({ }^{*} X\right)$.)

Call $\Omega$ well-distributed in $X$ provided that whenever $\left\{K_{n}\right\}$ is a sequence of compact sets with $m\left(K_{n}\right) \rightarrow 1, L(\mu)\left(\Omega \cap^{*} K_{n}\right) \rightarrow 1$. Every Radon space $(X, \mathscr{B}, m)$ has at least one well-distributed preimage, namely $\left({ }^{*} X, L\left({ }^{*} \mathscr{B}\right)\right.$, $\left.L\left({ }^{*} m\right)\right)$. On the other hand many interesting preimages are not well distributed, e.g., $\Omega=\{0, \Delta t, 2 \Delta t, \ldots, 1-\Delta t\}$ where $\Delta t=1 / H$ for some $H \in \in^{*} \mathbb{N}-\mathbb{N}$ and $\mu$ is normalized counting measure on $\Omega$.

6.1. Theorem. Consider the three statements:

(a) $f$ is strongly Lusin measurable,

(b) $f$ has a lifting,

(c) $f$ is Lusin measurable.

Then $(\mathrm{a}) \Rightarrow(\mathrm{b}) \Rightarrow(c)$. If, moreover, $\Omega$ is well distributed, then $(c) \Rightarrow(b)$. 
Proof. (a) $\Rightarrow$ (b) Let $\left\{K_{n}\right\}$ be a sequence of compact subsets of $X$ with $m\left(K_{n}\right) \rightarrow 1$, and $\left\{g_{n}\right\}$ continuous functions from $X$ to $Y$, such that $g_{n}=f$ on $K_{n}$. Let $A_{n} \subseteq\left(\Omega \cap \mathrm{st}^{-1} K_{n}\right)$ be internal such that $L(\mu)\left(A_{n}\right) \rightarrow 1$. By saturation there is an internal $F: \Omega \rightarrow{ }^{*} Y$ such that $F={ }^{*} g_{n}$ on $A_{n}$ for each $n \in \mathbb{N}$. If $\omega \in \bigcup_{n} A_{n}$, say $\omega \in A_{n}$, then ${ }^{\circ} \omega \in K_{n}$ and $F(\omega)={ }^{*} g_{n}(\omega) \approx$ $g_{n}\left({ }^{\circ} \omega\right)=f\left({ }^{\circ} \omega\right)$; it follows that $F$ is a two-legged lifting of $f$.

(b $\Rightarrow$ c) Suppose $F$ lifts $f$, i.e. $F(\omega) \approx f\left({ }^{\circ} \omega\right)$ for all $\omega$ in some set $\Omega^{\prime}$ with $L(\mu)\left(\Omega^{\prime}\right)=1$. Let $K_{0} \subseteq K_{1} \subseteq \cdots \subseteq X$ be compact with $m\left(K_{n}\right)>1-\frac{1}{n}$, let $A_{n} \subseteq\left(\Omega^{\prime} \cap \mathrm{st}^{-1} K_{n}\right)$ be internal with $\mu\left(A_{n}\right)>1-\frac{1}{n}$, let $F_{n}=\left\{(\omega, F(\omega)): \omega \in A_{n}\right\} \subseteq N S\left({ }^{*} X \otimes \otimes^{*} Y\right)$, let $G_{n}=\operatorname{st}\left(F_{n}\right)$, and let $E_{n}=\operatorname{proj}_{X} G_{n}=\operatorname{st}\left(A_{n}\right)$. Since $F_{n}$ is an internal subset of $N S\left({ }^{*} X \otimes^{*} Y\right), E_{n}$ and $G_{n}$ are closed. Since $E_{n} \subseteq K_{n}, E_{n}$ is compact. Clearly $G_{n} \subseteq \Gamma(f)$, so in fact $G_{n}$ must be compact as well; it follows that the restriction of $f$ to $E_{n}$ is continuous. Moreover, $m\left(E_{n}\right)=L(\mu)\left(\mathrm{st}^{-1} E_{n}\right) \geq L(\mu)\left(A_{n}\right)>1-\frac{1}{n}$; this proves (b).

(c $\Rightarrow \mathrm{b}$ ) Assume $\Omega$ is well distributed and $f$ is Lusin measurable. Proceed as in the proof of (c $\Rightarrow$ a), except (i) $\left\{g_{n}\right\}$ and $\left\{K_{n}\right\}$ need only satisfy that $g_{n}$ is continuous on $K_{n}$ (and not necessarily on all of $X$ ), and (ii) let $A_{n}=$ ${ }^{*} K_{n} \cap \Omega$. Well-distribution ensures that $L(\mu)\left(A_{n}\right) \rightarrow 1$, and the rest of the argument goes through as before.

This connection between Lusin measurability and two-legged liftings has useful consequences in each direction. For example, let $(\Omega, L(\mathscr{A}), L(\mu))$ be $\left({ }^{*} X, L\left({ }^{*} \mathscr{B}\right), L\left({ }^{*} m\right)\right)$ and $Y$ be second countable; then Theorem 6.1 and Theorem 4.1 imply

6.2. Corollary (Lusin's Theorem). Let $(X, \mathscr{B}, m)$ be Radon and $Y$ second countable; then every measurable function $f: X \rightarrow Y$ is Lusin measurable.

Observe that if $Y=\mathbb{R}$ then (by Tietze's extension theorem) Lusin measurable $=$ strongly Lusin measurable.

Note also that this proof of Lusin's Theorem is much simpler than it looks, as it only uses a special case of Theorem 4.1 (namely Anderson's theorem, which by itself has a fairly simple proof), together with the self-contained (b) $\Rightarrow(c)$ part of Theorem 6.1.

In the other direction, it is easy to find example (see [F, Remarks 3D and Example 3F]) of Radon $(X, \mathscr{B}, m)$, Hausdorff $Y$, and measurable $f: X \rightarrow$ $Y$ with $f$ not Lusin measurable; since each Radon probability has a Loeb preimage, it follows:

6.3. Corollary. There is a Loeb space $(\Omega, L(\mathscr{A}), L(\mu))$, a Hausdorff $Y$ with $2^{\operatorname{card}(Y)}<\kappa_{0}$, and a measurable function $f$ from $\Omega$ to $Y$, such that $f$ has no lifting.

The following example, modified from ([F, Remarks $3 \mathrm{D}]$ ), is a direct construction of a Loeb measurable function without a lifting. 
6.4. Example. Let $(\Omega, L(\mathscr{A}), L(\mu))$ be any Loeb preimage of Lebesgue measure $m$ on the interval $[0,1]$, let $Y$ be $[0,1]$ endowed with the "half-open" topology generated by sets of the form $[a, b)$, and let $f: \Omega \rightarrow Y$ be the standard part map with respect to the usual topology on $[0,1]$. Since the two topologies on $[0,1]$ give the same Borel sets, and the standard part map is measurable, $f$ is measurable.

Suppose $F: \Omega \rightarrow^{*} Y$ and $E \in \mathscr{A}$ are internal, with $F(\omega) \approx f(\omega)$ (in the "half-open" sense) for each $\omega \in E$. In particular, $F(E) \subseteq N S\left({ }^{*} Y\right)=$ $\left\{y \in{ }^{*}[0,1]: y \geq \operatorname{st}(y)\right\}$, where $\operatorname{st}(y)$ is the "usual" standard part of $y$. Put $E^{\prime}=\operatorname{st}(F(E))=\operatorname{st}(E)$. If $y \in(0,1], z \in{ }^{*}[0, y)$, and $y-z \approx 0$, then $(z, y) \cap F(E)=\phi$. By saturation there is some standard $z=z_{y} \in[0, y)$ with ${ }^{*}(z, y) \cap F(E)=\phi$. It follows that $(z, y) \cap E^{\prime}=\phi$, and $\left\{\left(z_{y}, y\right): y \in E^{\prime}\right\}$ is a collection of disjoint open intervals. This means that $E^{\prime}$ is countable, so $L(\mu)(E) \leq L(\mu)\left(\mathrm{st}^{-1}\left(E^{\prime}\right)\right)=m\left(E^{\prime}\right)=0 . F$, therefore, cannot be a lifting of $f$.

The final corollary is an entirely standard result which demonstrates the strength of the machinery developed in this paper. Call a set $E \subseteq X \otimes Y$ almost Baire provided for some $D \in \mathscr{S}(\mathscr{Z}), m\left(\operatorname{proj}_{X}(D \Delta E)\right)=0$, where $\mathscr{Z}$ is the paving of zero sets of continuous real-valued functions on $X \otimes Y$.

6.5. Corollary. Let $(X, \mathscr{B}, m)$ be Radon and suppose $\Gamma(f)$ is almost Baire; then $f$ is Lusin measurable.

Proof. Let $(\Omega, L(\mathscr{A}), L(\mu))=\left({ }^{*} X, L\left({ }^{*} \mathscr{B}\right), L\left({ }^{*} m\right)\right)$, and $f^{\prime}=f \circ$ st. Find $D \in \mathscr{S}(\mathscr{Z})$ with $m(A)=0$, where $A=\operatorname{proj}_{\Omega}(D \Delta \Gamma(f))$. Let $\left\{h_{\tau}: \tau\right.$ a finite sequence from $\mathbb{N}\}$ be a family of continuous functions from $X \otimes Y$ to $[0,1)$ such that $D=\cup \cap D_{\sigma \mid n}$, where $D_{\tau}=h_{\tau}^{-1}(0)$. Let $G=\mathrm{st}^{-1} D=\cup \cap \mathrm{st}^{-1} D_{\sigma / n}$; as in $[\mathrm{H}], \mathrm{st}^{-1} D_{\tau} \in \mathscr{S}\left(\mathscr{K}_{0}\right)$, so $G \in \mathscr{S}\left(\mathscr{K}_{0}\right)$.

Suppose $(\omega, y) \in \Omega \otimes^{*} Y$, and ${ }^{\circ} \omega \notin A$. The following four statements are evidently equivalent: (i) $(\omega, y) \in G$; (ii) $\left({ }^{\circ} \omega,{ }^{\circ} y\right) \in D$; (iii) $y \approx f\left({ }^{\circ} \omega\right)=$ $f^{\prime}(\omega)$; and (iv) $(\omega, y) \in \Gamma^{\circ}\left(f^{\prime}\right)$. It follows that $\operatorname{proj}_{\Omega}\left(G \Delta \Gamma^{\circ}\left(f^{\prime}\right)\right) \subseteq \mathrm{st}^{-1} A$. On the other hand, it is easy to see that $\Omega-\operatorname{proj}_{\Omega} G \subseteq \mathrm{st}^{-1}(A) \cup N S\left({ }^{*} X\right)^{c}$. Since $L(\mu)$ is a preimage of $m, L(\mu)\left(\mathrm{st}^{-1} A \cup N S\left({ }^{*} X\right)^{c}\right)=L(\mu)\left(\mathrm{st}^{-1} A\right)=m(A)=0$. By (c) $\Rightarrow$ (a) of Theorem 3.1, $f^{\prime}$ has a lifting, so by Theorem $6.1 f$ is Lusin measurable.

\section{FINAL REMARKS}

The first use of Choquet's Theorem 2.2 in the nonstandard setting was made by the author in 1987, in order to answer a question posed by Henson concerning the descriptive theory of analytic sets [RH]. This 'capacity trick' has since shown signs of becoming a standard tool in that area (e.g., [Z]).

The idea of using this theorem to construct liftings was suggested by the study 
of random sets. The problem of finding a lifting is essentially that of finding a certain kind of measurable selection, and Choquet's theorem is the standard tool for finding a measurable selection of a random closed set.

A different theorem of Choquet, also involving capacities, is given a nonstandard proof in [R1] and extended in [R2].

\section{ACKNOWLEDGMENTS}

I am indebted to Tom Lindstrom, for my interest in the general question; Keith Stroyan, my host in Iowa where I first developed the nonstandard capacity theory of which $\S 2$ forms a small part; Ward Henson, who recently revived my interest in capacity methods; and Nigel Cutland, for making possible my stay in Hull.

I am also grateful to the referee for his careful reading of the paper.

\section{REFERENCES}

[AFHL] S. Albeverio, J. E. Fenstad, R Hoegh-Krohn, and T. Lindstrom, Nonstandard methods in stochastic analysis and mathematical physics, Academic Press, New York, 1986.

[A] R. M. Anderson, Star-finite representation of measure spaces, Trans. Amer. Math. Soc. 271 (1982), 667-687.

[C1] N. J. Cutland, Nonstandard measure theory and its applications, Bull. London Math. Soc. 15 (1983), 529-589.

[C2] N. J. Cutland, (ed), Nonstandard analysis and its applications, Cambridge University Press, Cambridge, 1988.

[DM] C. Dellacherie and P. A. M. Meyer, Probabilities and potential, Hermann, 1978.

[F] D. H. Fremlin, Measurable functions and almost-continuous functions, Manuscripta Math. 33 (1981), 387-405.

[H] C. W. Henson, Analytic sets, Baire sets and the standard part map, Canad. J. Math. XXXI (1979), 663-672.

[HL] A. E. Hurd and P. A. Loeb, An introduction to nonstandard real analysis, Academic Press, New York, 1985.

[K] H.J. Keisler, An infinitesimal approach to stochastic analysis, Mem. Amer. Math. Soc. 297 (1984).

[L] P. A. Loeb, Conversion from nonstandard to standard measure spaces and applications in probability theory, Trans. Amer. Math. Soc. 211 (1975), 113-122.

[LR] D. Landers and L. Rogge, Universal Loeb-measurability of sets and of the standard part map with applications, Trans. Amer. Math. Soc. 304 (1987), 229-243.

[R1] D. Ross, Measurable transformations in saturated models of analysis, Ph. D. thesis, Madison, 1983.

[R2] - Random sets without separability, Ann. Probab. 14 (1986), 1064-1069.

[RH] D. Ross and C. W. Henson, Analytic mappings of hyperfinite sets (to appear).

[SB] K. D. Stroyan and J. M. Bayod, Foundations of infinitesimal stochastic analysis, North-Holland, Amsterdam, 1986.

[Z] B. Zivaljevic, Every Borel function is monotone Borel (to appear).

Department of Mathematics and Statistics, University of Minnesota, Duluth, MinNESOTA 55812 